\title{
Fathers experienced stillbirth as a waste of life and needed to protect their partners and express grief in their own way
}

Samuelsson M, Radestad I, Segesten K. A waste of life: fathers'experience of losing a child before birth. Birth 2001 Jun;28:124-30.

QUESTION: How do fathers experience the intrauterine death of their child?

Design

Phenomenology.

\section{Setting}

Sweden.

\section{Participants}

11 fathers (age range 31-46 y) whose partners had stillborn children at 32-42 weeks of pregnancy in 1997 and 1998. 6 fathers already had children. 4 fathers had become fathers again after the stillbirth, and 4 of the men's partners were pregnant at the time of the study.

\section{Methods}

Fathers were interviewed 5-27 months after the stillbirth in the venue of their choice (at home or the hospital). Interviews of 25 minutes to 2 hours in length were taped and transcribed. The transcripts were analysed to identify meaningful central units.

\section{Main findings}

(1) Experiencing that the baby was no longer alive: 8 fathers had a premonition that something was wrong before being told about the stillbirth, but they still had hope. Fathers described feelings of impending catastrophe (including shock, a lack of feeling, and denial). They also described great disappointment when their preparations and expectations for the baby were thwarted. Most fathers found consolation in their adversity through confirmation of their reactions by support and information. They expressed an urgent need to get rid of the burden, and were concerned that the dead baby could harm their partner. They initially felt that delivery should be by a caesarean section, but after receiving information on this were able to accept a vaginal delivery. (2) Time between being told of the baby's death and the induction of labour: fathers felt that having time to adjust to the baby's death and to calm down in peace and quiet was important. They wanted to take care of practical details and protect their partner, but found it difficult when they were distressed themselves. (3) The delivery: frustration and helplessness were common feelings experienced by the fathers during labour and delivery. They felt they could do little, and everything was focused on the physical aspect of their partner giving birth. Most fathers said that it felt right that their partner should give birth vaginally, despite their initial wish for a caesarean section. Fathers found reassurance in the care routine, and were generally satisfied with the process and felt supported, especially by the hospital chaplain and deaconesses. (4) The stillbirth and aftercare: fathers felt emptiness and silence at the moment of stillbirth. They felt sad about the waste of life. Fathers approached the baby with respect and dignity, and also with a fear of seeing their child. Not all fathers spontaneously approached the child and some needed persuasion and assistance. Fathers had tokens of remembrance from the baby including photos, hand and footprints, a lock of hair, and identification wrist bands. They found the concrete items helpful and felt they might not have chosen these if they had been left to themselves after the delivery. (5) The first time at home: feelings of emptiness and silence were pronounced when they went home - there were 3 of them when they left for the hospital but only 2 came back. Collected tokens of remembrance of the child and the support of the obstetric staff and hospital chaplain helped with finding their way back to everyday life. They thought that receiving support in their grief was an urgent need. Fathers often felt their partners needed to talk about their feelings more, whereas they were more reticent.

\section{Conclusions}

Fathers experienced the stillbirth of their baby as a waste of life. They felt inadequate and helpless during the actual delivery. They needed to protect their partner and grieve in their own way. Tokens of remembrance of the child were especially helpful, as was the support of the hospital chapel personnel.

\section{COMMENTARY}

The qualitative study by Samuelsson et al makes an important contribution toward our understanding of how traumatic it is for fathers as well as mothers to lose a child before birth. For obvious reasons, the mother of the child who is undergoing the labour and delivery is the focus of nursing care and attention. However, this interesting study provides us with an understanding of the father's experience and how nurses can help to respond to the father's needs as well as to the mother's. Fathers have needs for information and participation before, during, and after the delivery of a stillborn child. In their study, Samuelsson $e t$ al found that fathers seemed particularly appreciative of the tokens of remembrance of the baby collected by the staff, and admitted that at the time they would not have collected these of their own free will because they were not psychologically capable of realising their value in the future. Other suggestions made by the fathers included staff breaking the frightening silence before and during the revelation of the death of the baby, avoiding using medicotechnical terms, speaking to both parents at the time, encouraging parents to be together with the child after the delivery, supporting the father in helping his partner cope with her grief as well as his own, and recognising the father as a mourner. Fathers are likely to need extra support and encouragement from nurses to express their loss and grief.

11 of 20 potential participants agreed to be interviewed, leaving some question about whether the 9 non-participants would have shared similar experiences or whether they would have had unique experiences. However, the data from the 11 participants provide a rich guide to how nurses can ensure that fathers are not "forgotten mourners".

Cynthia Kitson, RN(EC), MN Primary Health Care Nurse Practitioner Pinecrest Queensway Health and Community Services Ottawa, Ontario, Canada 